\title{
Light scattering by particles with small-scale surface roughness: comparison of four classes of model geometries
}

\author{
Michael Kahnerta,b, Timo Nousiainen ${ }^{\mathrm{c}}$, Manu Anna Thomas ${ }^{\mathrm{a}}$, Jani \\ Tyyneläc \\ ${ }^{a}$ Swedish Meteorological and Hydrological Institute, Folkborgsvägen 1, SE-601 76 \\ Norrköping, Sweden \\ ${ }^{b}$ Chalmers University of Technology, Department of Earth and Space Science, SE-412 96 \\ Gothenburg, Sweden \\ ${ }^{c}$ Department of Physics, P. O. Box 48, FI-00014 University of Helsinki, Finland
}

\begin{abstract}
We compare four different model geometries for particles with small-scale surface roughness. The geometries are based on regular and stochastic surface perturbations, as well as on 2D- and 3D-roughness models. We further compare T-matrix and discrete dipole computations. Particle size parameters of 5 and 50 are considered, as well as refractive indices of $1.6+0.0005 \mathrm{i}$ and $3+0.1$ i. The effect of small-scale surface roughness on the intensity and polarisation of the scattered light strongly depends on the size parameter and refractive index. In general, 2D surface roughness models predict stronger effects than 3D models. Stochastic surface roughness models tend to predict the strongest depolarising effects, while regular surface roughness models can have a stronger effect on the angular distribution of the scattered intensity. Computations with the discrete dipole approximation only cover a limited range of size parameters. T-matrix computations allow us to significantly extend that range, but at the price of restricting the model particles to symmetric surface perturbations with small amplitudes.
\end{abstract}

Keywords: Scattering, aerosols, T-matrix, surface roughness, mineral dust, lidar

Email address: michael.kahnert@smhi.se (Michael Kahnert) 


\section{Introduction}

Small-scale surface roughness is a morphological feature that is found in many different types of particles in nature, such as mineral aerosol particles in planetary atmospheres, cosmic dust particles, regolith particles on the surface of terrestrial planets and asteroids, and ice-cloud particles rimed with supercooled water droplets. The impact of small-scale surface roughness on the scattered intensity and polarisation can, in some cases, even dominate over the effect of the particles' overall non-spherical shape. This is particularly the case for particles with high absorption cross sections $[1,2]$ and for particles composed of optically hard material [2].

Computing the optical properties of such particles is among the most challenging problems in electromagnetic scattering research. The roughness features are often smaller than the wavelength of light, so that the light scattering problem cannot be solved by use of the geometric optics approximation. On the other hand, the size parameter $x=2 \pi r / \lambda$ of the particles is often large compared to unity. (Here, $r$ denotes the volume-equivalent radius of the particle, and $\lambda$ is the wavelength of light.) For particles with large $x$, numerically exact solutions of Maxwell's equations are often plagued by ill-conditioning problems or high CPU-time requirements. Owing to these numerical limitations, very little is known about the significance of smallscale surface roughness for the optical properties of particles of different size parameters, morphologies, and chemical compositions. Studies for particles with large size parameters are particularly rare. A ray optics approximation (ROA) modified with partial Lambertian reflection/refraction has been used by [3] and [4], and another ROA model incorporated a radiative transfer approach [5], to study the impact of small-scale surface roughness for dust particles much larger than the wavelength. The former model was rather phenomenological and the latter assumed roughness elements to be independent scatterers, so neither model can be expected to provide fully accurate and reliable predictions for the impact of roughness.

Most theoretical studies have been limited to high-order, spherical 2DChebyshev particles $[1,6,2]$. More advanced model geometries involve spheroidal 2D-Chebyshev particles [1], spherical 3D-Chebyshev particles [7], Gaussian random particles [8, 9], and spheres covered by smaller spherical particles [10]. Investigations of 2D-Chebyshev particles revealed that the phase function initially changes and eventually converges as the order of the perturbing Chebyshev polynomial is increased. This indicates that the op- 
tical properties of particles are sensitive to the presence and the amplitude of a surface perturbation with a sub-wavelength scale, but they do not seem to depend on the detailed structure of that perturbation. This raises the question if it makes much of a difference how one chooses the representation of small-scale surface roughness in a model. For instance, does it matter if we employ particles with a 2D- or a 3D-surface roughness? Does it make a difference if our model particles have a regular or a stochastic roughness structure?

Due to numerical constraints, almost all studies of particles with smallscale surface roughness have either been limited to size parameters of $x \lesssim 15$ or have been based on non-physical parameterisations or independent multiple scattering treatments that leave out part of the physics involved. Recent work reported in [7] was based on numerically exact T-matrix calculations for 3D-Chebyshev particles with size parameters up to $x=70$. This substantial increase in the range of size parameters has been achieved by a numerical approach that makes combined use of group theoretical methods [11, 12] and a perturbation formulation [13] of the null-field method. The perturbation approach reduces numerical ill-conditioning problems, but it is limited to surface perturbations with small amplitudes. The group theoretical approach reduces CPU-time by 4-6 orders of magnitude, depending on particle size and on the order of the perturbing polynomial. However, it is limited to particles with symmetric surface perturbations and base geometries. Despite these limitations, the method offers the potential for investigating the effects of small-scale surface roughness for a significantly extended range of size parameters by use of numerical methods based on rigorous electromagnetic scattering theory.

The studies in $[1,6,2,7,10]$ were based on T-matrix computations with the null-field method, while [8,9] made use of the discrete dipole approximation (DDA). Exploiting geometric symmetries and formulating a perturbation approach for small-scale surface roughness is relatively straightforward in the null-field method and in other T-matrix methods. Also, optical properties of randomly oriented particles can be computed analytically with higher accuracy and efficiency $[14,15]$. However, the null-field method is, in general, limited to star-shaped particles that do not deviate too strongly from spherical shape, and it is mostly applied to homogeneous particles. The DDA, on the other hand, is a highly flexible method that has no such limitations. However, the DDA is a computationally demanding method that does not lend itself easily to exploiting geometric symmetries, and it requires time- 
consuming numerical orientation-averaging. Thus, either method offers specific advantages for computing light scattering by particles with small-scale surface roughness.

This short review of recent work on particles with small-scale surface roughness leaves several questions unanswered, of which we will address the following ones in the present study.

- What are the differences and similarities of particles with a 2D- and 3Dsurface roughness, and of particles with regular and stochastic surface perturbations? In which optical properties do the differences (if any) manifest themselves, and how does the comparison of different surfaceroughness models depend on the size parameter and the refractive index of the particles?

- What are the strengths and limitations of the null-field method and the discrete dipole approximation in applications to particles with smallscale surface roughness? What are the main sources of error in either method, and what is the range of size parameters that can be covered for particles with irregular, small-scale surface roughness?

To keep this work computationally manageable, we will limit our study to mineral particles. The details on the selection of our case studies, model geometries, and numerical methods are given in Sect. 2. Computational results are presented in Sect. 3 and discussed in Sect. 4. Concluding remarks are given in Sect. 5 .

\section{Methods}

The aim of this study is two fold. First, we want to investigate the suitability of both the T-matrix approach and the discrete dipole approximation (DDA) for particles with small-scale surface roughness. Second, we want to compare the optical properties computed with different model geometries for small-scale surface roughness with varying degree of sophistication and computational complexity.

\subsection{Computational methods}

We compute the T-matrix by the use of Waterman's null-field method [16], also known as the extended boundary condition method. The advantages of this method are that the computation of orientation-averaged 
optical properties can be performed analytically [14, 15], and that particle symmetries can be systematically exploited $[12,17]$. A disadvantage is that the method can become numerically ill-conditioned for particles that deviate strongly from spherical shape, for particles that have a large real or imaginary part of the refractive index, and for nonspherical particles that are much larger than the wavelength of light. Ill-conditioning problems are also quite common for particles with small-scale surface roughness, which restricts applications of the null-field method to rough particles with size parameters up to about $10-15$ (e.g. $[2,7]$ ), and to small roughness amplitudes. This range can be considerably extended for particles with a symmetric small-scale surface roughness by combining group theory with a perturbation approach [7]. For example, for 3D Chebyshev particles with a refractive index of $m=3+0.1 \mathrm{i}$, conventional T-matrix computations give accurate results for size parameters up to 14 , while the perturbative approach is numerically stable for size parameters up to 70 [7], thus extending the range of size parameters by a factor of 5. At the same time, exploiting symmetries by the use of group theory reduces computation time for such particles by 4-6 orders of magnitude (depending on particle size and on the order of the symmetry group), and it reduces the scaling of CPU-time with size parameter from $\sim x^{5.5}$ (without symmetries) to $\sim x^{3.5}$ (with symmetries) [7]. Thus, at least for particles with symmetric small-scale roughness structures, the applicability range of the T-matrix approach can be substantially extended.

The discrete dipole approximation is a volume-integral equation method. The main advantage is that it is, in principle, applicable to any kind of model geometry. There are no limitations to homogeneous or only slightly nonspherical particles. However, its CPU-time requirements are high and strongly increase with size parameter, which practically limits this method to particles up to size parameters of about 10-15 with orientation averaging. Capturing the effect of small-scale morphological features may require a very fine grid in the discretisation of the volume-integral term, which can increase the computation time even more. Also, averaging over orientational angles needs to be performed numerically. This means that the scattering problem needs to be solved repeatedly for a sufficiently large set of discrete orientational angles.

For T-matrix computations we use the program Tsym [11, 12], which has been specifically tailored to exploiting group theory in T-matrix computations. The perturbation T-matrix method has recently been implemented and tested in the model [7]. For the DDA-computations, we use the programs 

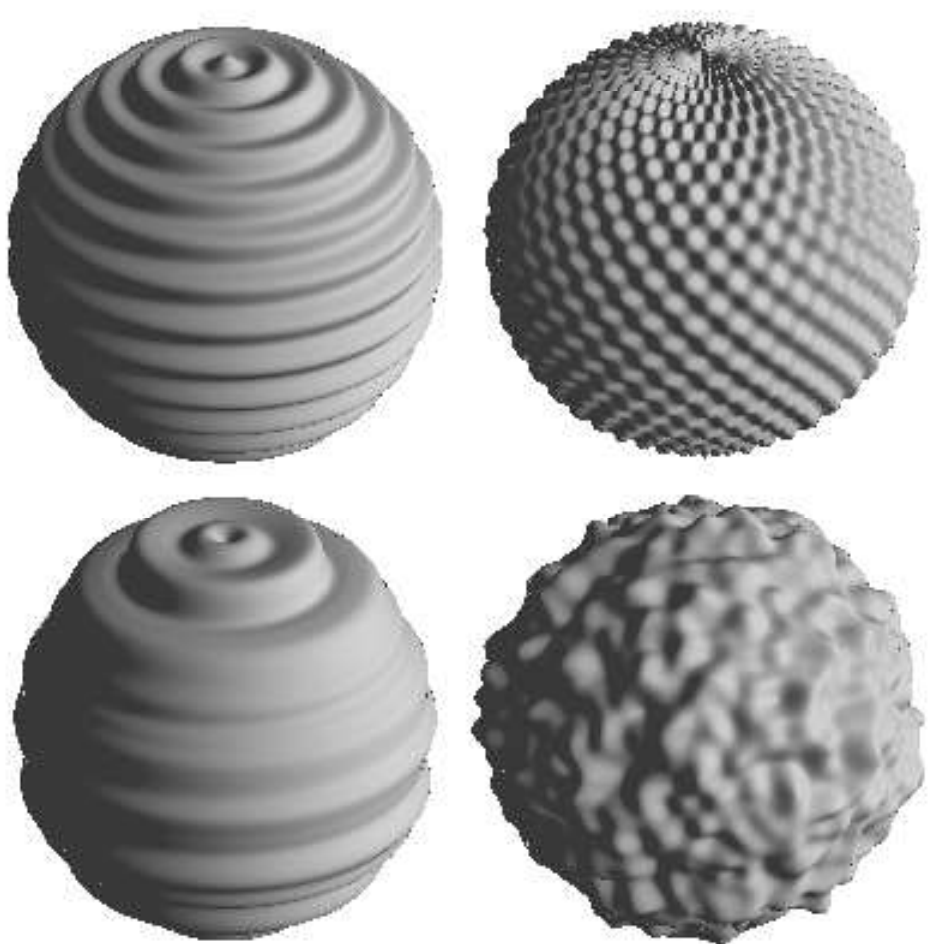

Figure 1: Model particles considered in this study: 2D-Chebyshev (upper left), 3DChebyshev (upper right), 2D-Gaussian random spheres (lower left), and 3D-Gaussian random spheres (lower right).

$D D S C A T$ (e.g. [18]) and $A D D A$ (e.g. [19]). These two models have recently been cross-validated in a model intercomparison study [20]. The accuracy of the T-matrix results is cross-checked by use of the reciprocity condition (e.g. $[13,7])$. These tests involve the computation of the T-matrix for particles in at least two different fixed orientations, and comparing the polarised differential scattering cross sections. These computations are numerically very demanding for irregular particles. The reciprocity tests were therefore not performed for 3D Gaussian random spheres. We require that the reciprocity condition is fulfilled with an error of less than $5 \%$. 


\subsection{Model particles}

We investigate the differences in the optical properties of particles with $2 \mathrm{D}$ and 3D surface roughness, and with regular and stochastic surface roughness. To this end, we consider four different classes of model particles, which are illustrated in Fig. 1.

1. 2D Chebyshev particles (Cheb-2D, upper left): This is the simplest of all four classes of model particles. The surface parametrisation is given by

$$
r(\theta)=r_{0}[1+\epsilon \cos (\ell \theta)]
$$

where $r_{0}$ denotes the radius of the unperturbed sphere, $\theta$ is the polar angle, $\ell$ is the order of the Chebyshev polynomial, and $\epsilon$ represents the deformation parameter. The surface of these particles is fully characterized by the pair $(\theta, r(\theta))$. This is why we refer to particles with such an axisymmetric surface perturbation as particles with a "2D surface roughness".

2. 3D Chebyshev particles (Cheb-3D, upper right): The surface parametrisation of these particles is given by

$$
r(\theta, \phi)=r_{0}[1+\epsilon \cos (\ell \theta) \cos (\ell \phi)]
$$

where $\phi$ is the azimuth angle. So, the surface is characterised by $(\theta, \phi, r(\theta, \phi))$. We refer to any kind of non-axisymmetric surface perturbation as "3D surface roughness".

3. 2D Gaussian random spheres (GRS-2D, lower left): Just like Cheb-2D, GRS-2D geometries are only perturbed in the polar direction. Thus they have a higher symmetry than the Cheb-3D particles. However, in contrast to 2D- and 3D Chebyshev particles, the GRS-2D are not perturbed by a polynomial with a regular oscillation period and constant amplitude. Rather, the surface is stochastically perturbed with a prescribed amplitude standard deviation $\sigma$ and angular correlation $\Gamma$ [21]. Thus, the surface roughness of these particles is stochastic in the polar direction, although the geometry is highly symmetric owing to the axial symmetry.

4. 3D Gaussian random spheres (GRS-3D, lower right): This is the most complex and computationally most demanding class of model particles. It is perturbed by a stochastic 3D surface roughness with prescribed amplitude standard deviation $\sigma$ and angular correlation $\Gamma[21]$. 
Cheb-2D and Cheb-3D are part of the standard implementation of Tsym. GRS-2D and GRS-3D have been added to the program by adapting the publicly available GRS code described in [21]. This code provides the surface parametrisation $r(\theta, \phi)$ for GRS-2D and GRS-3D. We made some minor amendments to the code in order to obtain, additionally, the partial derivatives $\partial r / \partial \theta$ and $\partial r / \partial \phi$, which are needed in the null-field method.

We perform computations for two different size parameters, $x=5$ and $x=50$. Computations for irregular GRS-3D particles are computationally very demanding. For those particles, we have therefore only performed computations for $x=5$. All computations have been performed for two different refractive indices, $m=1.6+0.0005 \mathrm{i}$ and $m=3+0.1 \mathrm{i}$. The first refractive index is taken as a typical value at visible wavelengths for terrestrial silicate particles with a low concentration of hematite. The second refractive index is representative for pure hematite particles at visible wavelengths (neglecting birefringence). Henceforth, we will label computational results obtained for $m=1.6+0.0005 i$ by "silicate", and those obtained for $m=3+0.1$ i by "hematite".

\section{Results}

\subsection{T-matrix - DDA comparison for GRS-3D}

In the DDA, the geometry is represented by a discretised grid of dipoles. The main challenge is therefore to choose sufficiently small dipole spacing capable of resolving small-scale surface roughness features. This introduces computational demands that are significantly higher than those in computations for particles with smooth boundary surfaces. Computations for the size parameter 50 would have required huge amount of computing power, especially with orientation averaging, and might have also had numerical convergence problems, so DDA simulations were only carried out for the size parameter of $x=5$. To assure that the surface roughness is accounted for in full detail in the simulations, the DDA target shapes included about 360000 dipoles.

Naively, one may expect that in the T-matrix approach there are no problems related to the representation of the geometry. This is, indeed, the case for Cheb-2D and Cheb-3D, but not necessarily for GRS-2D and GRS3D. For Chebyshev particles, we have closed-form expressions for both the surface parametrisation $r(\theta, \phi)$ and its partial derivatives. In contrast, for 
GRS the computation of $r(\theta, \phi)$ and its partial derivatives involves numerical recurrence relations for modified spherical Bessel functions, associated Legendre functions $P_{l}^{m}(z)$, and derivatives of associated Legendre functions $\mathrm{d} P_{l}^{m}(z) / \mathrm{d} z$. GRS particles of small-scale surface roughness have small angular correlations, which requires numerical computations of high orders and degrees of $P_{l}^{m}(z)$ and $\mathrm{d} P_{l}^{m}(z) / \mathrm{d} z$. In numerical computations, we may encounter error accumulations at higher orders and degrees of these functions. Further, the smaller the angular correlation, the larger the argument in the spherical Bessel functions (see [21] for details). However, for large arguments the recurrence relations for the spherical Bessel functions can become numerically inaccurate. Small numerical inaccuracies in the computations of $r(\theta, \phi)$ may not be a problem in DDA computations. After all, the geometries are stochastic, and minor imprecisions in the generation of the geometries are not expected to be a major problem. In contrast, in the null field method we need not only $r(\theta, \phi)$, but also $\partial r / \partial \theta$ and $\partial r / \partial \phi$. Numerical inaccuracies in the computation of these quantities may result in inconsistencies between the surface parametrisation and its partial derivatives, since these three quantities are computed independently from each other. This is a possible source of error in T-matrix computations that does not affect DDA computations.

We found that the numerical routines for computing the modified spherical Bessel functions fail to produce accurate results for angular correlations $\Gamma$ smaller than $4^{\circ}$. All computations for GRS-2D and GRS-3D have therefore been performed for an angular correlation of $\Gamma=4^{\circ}$. The accuracy of the T-matrix results has been tested by use of the reciprocity condition, as explained earlier.

Table 1: Comparison of optical properties of GRS-3D particles computed with Tsym, $D D S C A T$, and $A D D A$

\begin{tabular}{ll|llllll} 
material & program & $Q_{\text {ext }}$ & $\omega$ & $g$ & $Q_{\text {bak }} / \mathrm{sr}^{-1}$ & $\delta_{L}$ & $\delta_{C}$ \\
\hline Silicate & Tsym & $2.97(1)$ & $0.995(0)$ & $0.576(1)$ & $0.393(6)$ & $0.0004(1)$ & $0.0008(2)$ \\
Silicate & DDSCAT & 3.06 & 0.996 & 0.587 & 0.317 & 0.0005 & 0.0009 \\
Hematite & Tsym & $2.49(1)$ & $0.560(2)$ & $0.670(5)$ & $0.074(5)$ & $0.002(1)$ & $0.004(2)$ \\
Hematite & ADDA & 2.59 & 0.577 & 0.673 & 0.075 & 0.003 & 0.005
\end{tabular}

Table 1 presents computational results for the extinction efficiency $Q_{\text {ext }}$, the single-scattering albedo $\omega$, the asymmetry parameter $g$, the backscattering efficiency $Q_{\text {bak }}$, the linear depolarisation ratio $\delta_{L}$, and the circular 
depolarisation ratio $\delta_{C}$. All computations were performed for GRS-3D particles with a size parameter of $x=5$, an angular correlation of $\Gamma=4^{\circ}$ and a relative radial standard deviation of $\sigma=0.03$. The first two rows compare results for silicate GRS-3D obtained with Tsym and DDSCAT. The last two rows show a corresponding comparison for hematite GRS-3D obtained with Tsym and ADDA. ADDA employs the filtered coupled dipoles (FCD) scheme [22] for computing dipole polarizability; this scheme performs well for high-refractive-index targets. Indeed, $D D S C A T$ did not produce convergent results for the hematite particles with any of its three optional polarizability schemes.

The Tsym calculations were performed for ten stochastic realisations of GRS-3D geometries. The data entries in the table show the average results for the ten geometries and, in parenthesis, the maximum deviation of the last significant figure from the average value. (For instance, an entry of $0.560(2)$ means that the average value of 0.560 varies, in general, within a maximum range of $\left[0.560-\delta_{1}, 0.560+\delta_{2}\right]$, and $\max \left\{\delta_{1}, \delta_{2}\right\}=0.002$.) DDSCAT computations were performed for three different geometries. Here we present the results for that geometry that gave intermediate results for the exinction efficiency.

We can make two interesting observations here. First, the relative differences in optical properties among different stochastic realisations of the GRS-3D geometries are very small, except for $\delta_{L}$ and $\delta_{C}$. The same is true for the three stochastic geometries for which DDA computations have been performed (not shown). Second, the T-matrix and DDA results for $Q_{\text {ext }}$, $\omega, g$, and $Q_{\text {bak }}$ agree to within a few percent, while differences in $\delta_{L}$ and $\delta_{C}$ between the two numerical methods are comparable to differences among different stochastic realisations of the GRS geometry.

Figure 2 shows a comparison of the Mueller matrix elements $F_{11}$ (first row), $F_{22} / F_{11}$ (second row), and $-F_{12} / F_{11}$ (third row) as a function of the scattering angle, obtained for silicate (left column) and hematite (right column) GRS-3D. The maximum range of values obtained for the ten GRS-3D geometries for which T-matrix calculations were performed are presented (grey shading), and the results obtained for the three GRS-3D geometries for which the DDA computations were performed are represented by solid, dashed, and dash-dotted lines.

For silicate particles, differences among the ten geometries used in Tmatrix computations, and among the three geometries used in DDA compuatations are relatively small; T-matrix and DDA results are close to each 

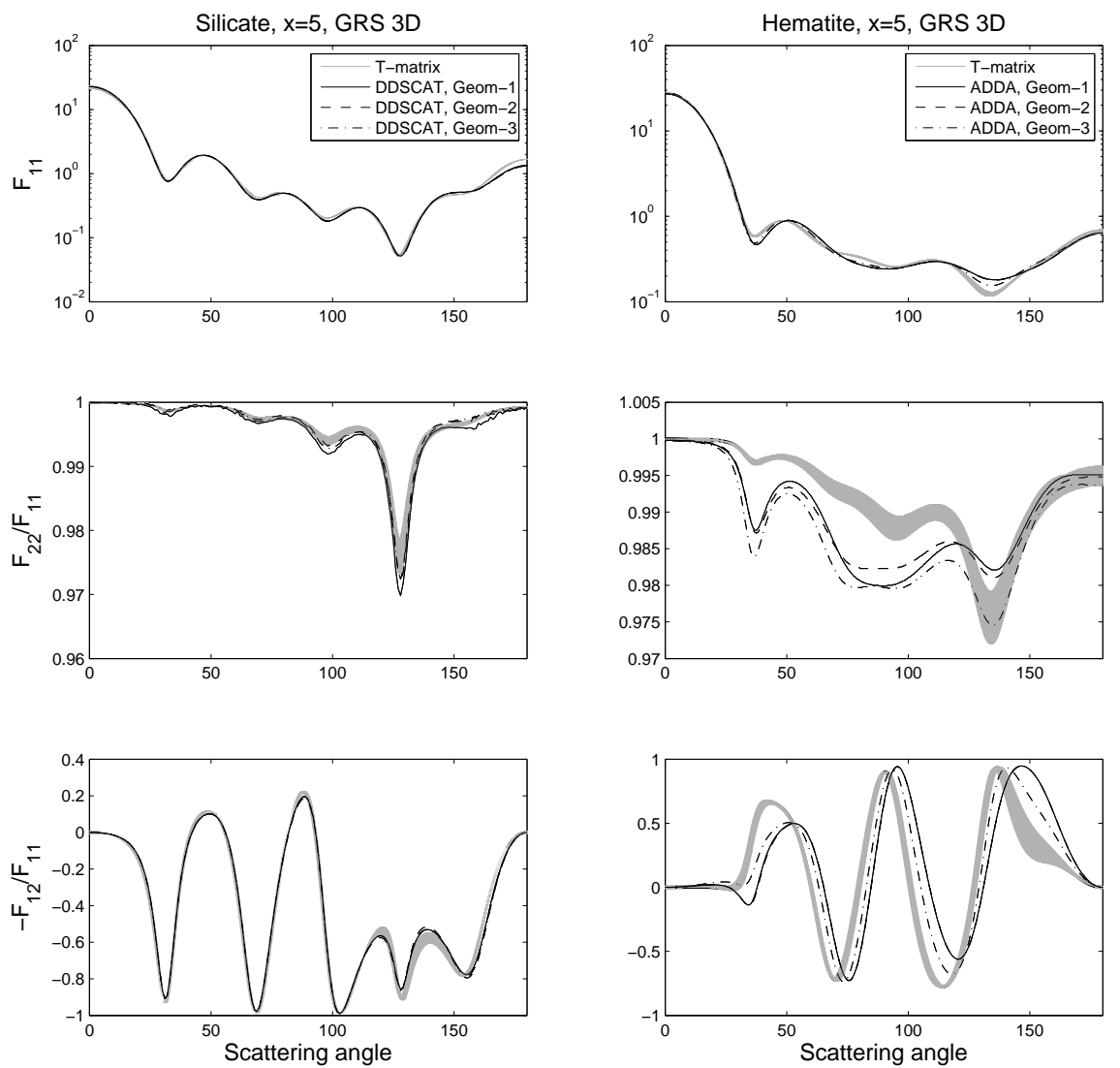

Figure 2: Mueller matrix elements $F_{11}$ (first row), $F_{22} / F_{11}$ (second row), and $-F_{12} / F_{11}$ of silicate (left column) and hematite particles (right column). All computations were performed for GRS-3D particles with $x=5$. The Tsym results (grey shading) indicate the maximum range covered by ten stochastic realisations of the GRS-3D geometry. DDSCAT and $A D D A$ computations each were performed for three stochastic realisations, represented by solid, dashed, and dash-dotted lines. 
other. For hematite, somewhat larger deviations among different GRS-3D geometries are observed, especially for $F_{22} / F_{11}$ and $-F_{12} / F_{11}$. Relative differences between Tsym and $A D D A$ results for hematite are significantly larger than those between Tsym and DDSCAT results for silicate. This is particularly apparent for $F_{22} / F_{11}$ at scattering angles of $20-120^{\circ}$. However, note that in either case, the deviations of $F_{22} / F_{11}$ from unity, and thus also the absolute errors, are small.

\subsection{Comparison of model particles}

Table 2: Comparison of optical properties of silicate particles represented by different model geometries. For $x=5, \epsilon=0.03$ (for Chebyshev particles) and $\sigma=0.03$ (for GRS particles). For $x=50, \epsilon=0.01, \sigma=0.01$. GRS results were averaged over ten stochastic geometries. All computations were performed with Tsym.

\begin{tabular}{ll|llllll} 
x & model & $Q_{\text {ext }}$ & $\omega$ & $g$ & $Q_{\text {bak }} / \mathrm{sr}^{-1}$ & $\delta_{L}$ & $\delta_{C}$ \\
\hline 5 & GRS-2D & $3.00(10)$ & $0.995(0)$ & $0.58(1)$ & $0.36(5)$ & $0.002(1)$ & $0.005(3)$ \\
5 & GRS-3D & $2.97(1)$ & $0.995(0)$ & $0.576(1)$ & $0.393(6)$ & $0.0004(1)$ & $0.0008(2)$ \\
5 & Cheb-2D & 2.96 & 0.995 & 0.575 & 0.407 & 0 & 0 \\
5 & Cheb-3D & 2.96 & 0.995 & 0.574 & 0.406 & 0 & 0 \\
5 & Spheres & 2.96 & 0.995 & 0.574 & 0.405 & 0 & 0 \\
& & & & & & & \\
50 & GRS-2D & $2.16(6)$ & $0.943(3)$ & $0.79(1)$ & $0.41(7)$ & $0.16(6)$ & $0.4(1)$ \\
50 & Cheb-2D & 2.15 & 0.955 & 0.82 & 0.58 & 0 & 0 \\
50 & Cheb-3D & 2.15 & 0.957 & 0.81 & 0.59 & 0 & 0 \\
50 & Spheres & 2.15 & 0.956 & 0.80 & 0.58 & 0 & 0
\end{tabular}

Table 2 presents a comparison of optical properties of silicate particles computed for size parameters $x=5$ and $x=50$ for different model particles. All computations were performed with the Tsym program. For GRS-2D and GRS-3D, an average over ten stochastic realisations of the geometry has been performed, where we assumed an angular correlation of $\Gamma=4^{\circ}$ and a relative amplitude standard deviation of $\sigma=0.03$ (for $x=5$ ) and $\sigma=0.01$ (for $x=50$ ). The data entries show the mean values and the maximum ranges of the last significant figures. For Cheb-2D and Cheb-3D particles, we show computational results for Chebyshev order of $\ell=30$ and deformation parameter $\epsilon=0.03$ (for $x=5$ ), and $\ell=240$ and $\epsilon=0.01$ (for $x=50$ ). The Chebyshev orders are chosen such that the perturbation wavelength $\Lambda=2 \pi r / \ell$ of the Chebyshev polynomial on the surface of a 
particle with radius $r$ is $\Lambda \lesssim \lambda / 4$, where $\lambda$ is the wavelength of light. This means that $x / \ell \lesssim 1 / 4$.

We can see that $\omega$, and $g$ vary very little among different model particles for both size parameters. In fact, the results obtained for GRS-2D, GRS-3D, Cheb-2D, and Cheb-3D are very close to those obtained for smooth spherical particles. Similarly, the values obtained for $Q_{\text {ext }}$ are very similar for all particles including spheres. However, note that the variability of $Q_{\text {ext }}$ for different stochastic realisations of GRS-2D geometries with $x=5$ is fairly high. Larger differences among various model particles are obtained for $Q_{\text {bak }}$. While Cheb-2D and Cheb-3D give backscattering efficiencies that are close to those for spheres, GRS-3D and, even more so, GRS-2D particles yield somewhat lower values of $Q_{\text {bak }}$. For $x=50, Q_{\text {bak }}$ computed with GRS-2D is almost $30 \%$ lower than the corresponding results obtained for spheres, Cheb2D, and Cheb-3D. Interestingly enough, both GRS-2D, GRS-3D predict nonzero linear and circular depolarizations, while Cheb-2D and Cheb-3D are non-depolarising, just like spheres. GRS-2D yield $\delta_{L}$ and $\delta_{C}$ values that are almost an order of magnitude larger than those computed with GRS-3D. Both $\delta_{L}$ and $\delta_{C}$ computed with GRS-2D are small for $x=5$, but rather substantial for $x=50$.

Figure 3 shows Mueller matrix elements computed with the different model particles for silicate particles with $x=5$ (left column) and $x=50$ (right column). The grey shading indicates the maximum range obtained for the ten GRS-2D model particles. The corresponding range for the ten GRS-3D particles is significantly smaller (not shown).

For $x=5$ the phase functions $F_{11}$ (upper left) of all model particles are almost identical. Also, there is very little variation among the ten GRS2D geometries. Significant differences are observed for $F_{22} / F_{11}$ (centre left), which is responsible for linear depolarisation. While spheres, Cheb-2D, and Cheb-3D all give $F_{22} / F_{11}=1$, thus zero depolarisation, for all scattering angles, GRS-2D display values of $F_{22} / F_{11}$ as low as $92 \%$. The ensembleaveraged results for GRS-3D are closer to unity as compared to those obtained for GRS-2D. The lowest values are observed around scattering angles of $130^{\circ}$. We also see that $F_{22} / F_{11}$ varies significantly among the ten GRS-2D geometries, with the largest differences occuring around $130^{\circ}$. The degree of linear polarisation $-F_{12} / F_{11}$ is rather similar for all particles including spheres, except in the angular range of $110-150^{\circ}$.

For $x=50$, the Mueller matrix elements computed for spheres (black line) are almost identical with those computed for Cheb-2D and Cheb-3D with 

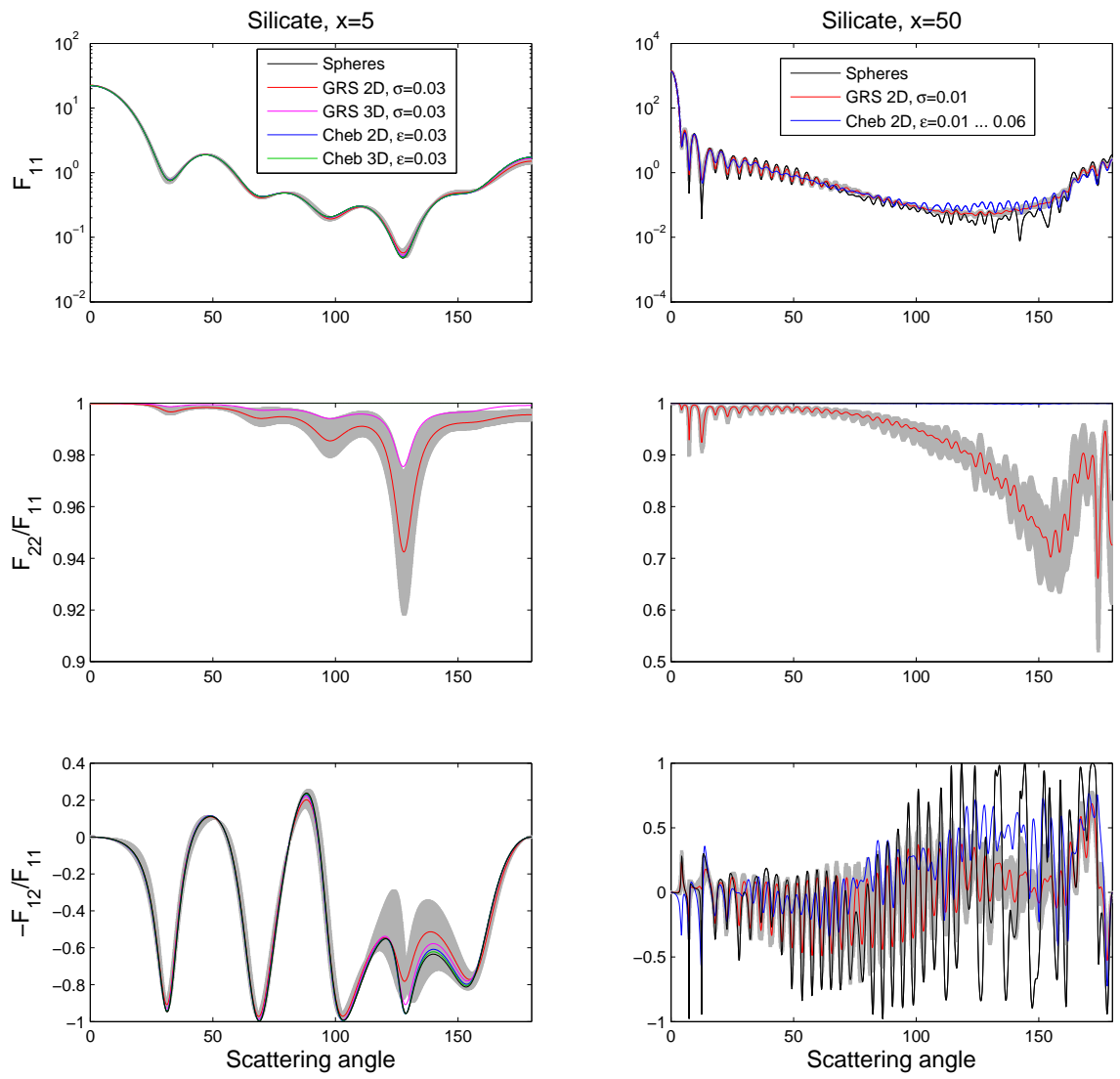

Figure 3: Mueller matrix elements $F_{11}$ (first row), $F_{22} / F_{11}$ (second row), and $-F_{12} / F_{11}$ (third row) of silicate particles with $x=5$ (left column) and $x=50$ (right column). All computations were performed with Tsym. The grey shading indicates the maximum range of ten stochastic realisations of the GRS-2D geometries. 
$\epsilon=0.01$. The latter two are therefore not shown. Instead, we performed computations for Cheb-2D with $\epsilon=0.01,0.02, \ldots, 0.06$ and computed an equi-probable average over these six Cheb-2D geometries (blue line). Also, we show GRS-2D results for $\sigma=0.01$. Very little differences are observed for the phase function (upper right). Even the extended range of deformation parameters used for the Cheb-2D geometries only produces minor differences in the angular range of $110-150^{\circ}$. The phase function obtained by averaging over ten GRS-2D geometries is smoother than that of spheres and displays less pronounced oscillations. The most striking differences are observed for $F_{22} / F_{11}$. For spheres, this quantity is always unity. For Cheb-2D the deviations from unity are so small that they are barely visible in the figure. By contrast, GRS-2D geometries deviate from unity by almost up to $50 \%$ and show considerable variability among the ten different geometries. We observe a broad local minimum aroung $150^{\circ}$, and a narrow double-lobe feature near the backscattering direction. The degree of linear polarisation $-F_{12} / F_{11}$ is characterised by a large number of oscillations, where the oscillation amplitude is largest for spheres and smallest for the ensemble-averaged GRS-2D geometries. Interestingly enough, the variations of $-F_{12} / F_{11}$ among the ten GRS-2D geometries are smaller than the oscillation amplitudes observed for spheres. This can be understood from the more regular structure of the internal electromagnetic field for spherical particles than for nonspherical particles, such as particles with GRS geometries [23].

Table 3: As Table 2, but for hematite particles.

\begin{tabular}{ll|llllll} 
x & model & $Q_{\text {ext }}$ & $\omega$ & $g$ & $Q_{\text {bak }} / \mathrm{sr}^{-1}$ & $\delta_{L}$ & $\delta_{C}$ \\
\hline 5 & GRS-2D & $2.51(5)$ & $0.562(5)$ & $0.674(8)$ & $0.06(2)$ & $0.006(14)$ & $0.01(3)$ \\
5 & GRS-3D & $2.49(1)$ & $0.560(2)$ & $0.675(3)$ & $0.074(5)$ & $0.002(1)$ & $0.005(2)$ \\
5 & Cheb-2D & 2,57 & 0.567 & 0.669 & 0.077 & 0.001 & 0.002 \\
5 & Cheb-3D & 2.43 & 0.567 & 0.661 & 0.092 & 0 & 0 \\
5 & Spheres & 2.43 & 0.572 & 0.656 & 0.095 & 0 & 0 \\
& & & & & & & \\
50 & GRS-2D & $2.14(2)$ & $0.624(0)$ & $0.803(1)$ & $0.020(0)$ & $0.0003(1)$ & $0.0006(2)$ \\
50 & Cheb-2D & 2.14 & 0.527 & 0.954 & 0.002 & 0 & 0 \\
50 & Cheb-3D & 2.14 & 0.572 & 0.883 & 0.009 & 0 & 0 \\
50 & Spheres & 2.14 & 0.625 & 0.803 & 0.020 & 0 & 0
\end{tabular}

Table 3 shows results analogous to those in Table 2, but for hematite 
particles. The effect of surface roughness on the optical properties is generally more pronounced for hematite than for silicate. For $x=5$ we observe that $Q_{\text {ext }}$ computed for different model particles can differ by up to $6 \%$ from that computed for spheres, while little variations are seen for $\omega$ and $g$. For $x=50$ the situation is reversed. While all model particles essentially yield identical results for $Q_{\text {ext }}$, significant differences are obtained for $\omega$ and $g$. Even more remarkable, $\omega$ and $g$ computed with either 2D or 3D Chebyshev particles deviate more from spheres than corresponding results computed with GRS2D. Thus $\omega$ and $g$ show a very different sensitivity to the choice of model geometry than $\delta_{L}, \delta_{C}$, and $F_{22} / F_{11}$, for which the deviation from spheres is most pronounced for GRS-2D.

In Fig. 4 , the Mueller matrix elements $F_{11}, F_{22} / F_{11}$, and $-F_{12} / F_{11}$ are compared for hematite spheres, GRS-2D, GRS-3D, Cheb-2D, and Cheb-3D. As for silicate, computations were performed for ten stochastic realisations of the GRS-2D geometry. The maximum range of the GRS-2D results is indicated by the grey shading, while the red line indicates the average over the ten different geometries. For $x=5$, the phase functions computed with different model particles and with different GRS-2D geometries display little variation, except in the backscattering region of $150-180^{\circ} . F_{22} / F_{11}$ varies significantly among GRS-2D geometries. The lowest values of about 0.92 are found at scattering angles around $130^{\circ}$. GRS-3D and Cheb-2D show qualitatively similar angular dependencies of $F_{22} / F_{11}$. But quantitatively it seems that stochastic surface roughness yields more depolarisation than regular roughness models, and $2 \mathrm{D}$ roughness models give stronger depolarisation than $3 \mathrm{D}$ roughness models. In fact, for Cheb-3D particles $F_{22} / F_{11}$ is identically equal to unity. The smallest deviations among the different model particles are seen in forward-scattering directions up to about $50^{\circ}$. The degree of linear polarisation $-F_{12} / F_{11}$ follows a similar pattern for all model geometries including spheres, but some deviations in the position, heights, and depths of the maxima and minima are evident.

The results obtained for $x=50$ are among the most striking. Unlike silicate, we observe substantial differences in the phase functions $F_{11}$ computed with different model particles. (Note that all phase functions are, in fact, properly normalised, which can be difficult to see on a logarithmic scale.) Spheres and GRS-2D yield almost identical results, while Cheb-3D and, even more so, Cheb-2D show strong departures from spheres. Both classes of Chebyshev particles scatter more anisotropically, i.e., they give more forward and less backscattering than spheres. This explains the large 

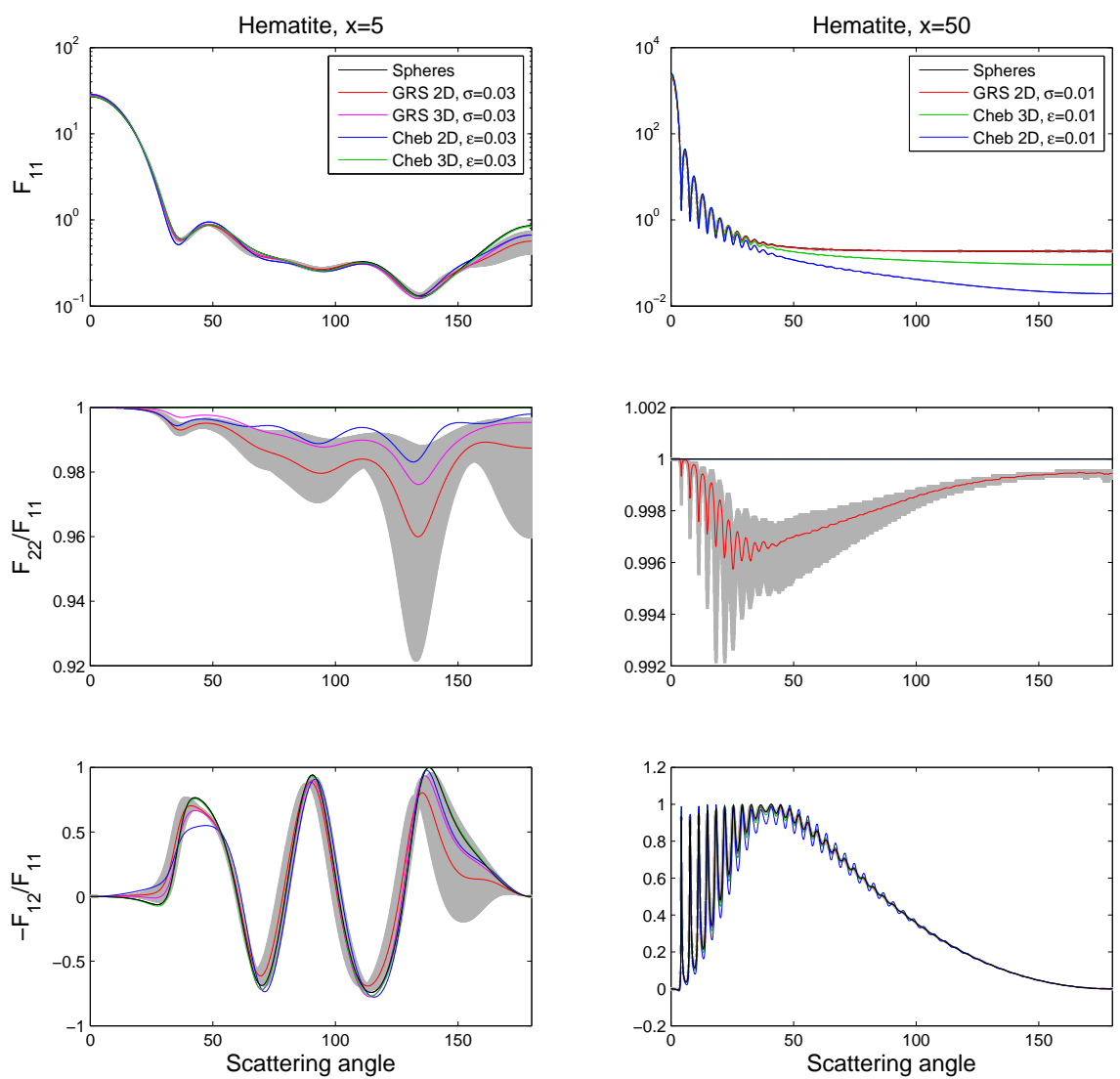

Figure 4: As Fig. 3, but for hematite particles. 
asymmetry parameters we found for Chebyshev particles at $x=50$ in Table 3. For $F_{22} / F_{11}$, the situation is reversed. While both Cheb-2D and Cheb$3 \mathrm{D}$ give results identically equal to unity (thus identical with the results for spheres), the GRS-2D geometries depart from unity. However, the departure from unity is smaller than for $x=5$. The reason is that for $x=5$ we considered relative standard deviations of $\sigma=0.03$, while for $x=50$ we were limited to $\sigma=0.01$ due to computational instabilities for larger surface-roughess amplitudes at large size parameters. Interestingly enough, for $x=50$ the lowest values of $F_{22} / F_{11}$ and the largest variations among the ten different GRS-2D geometries are now observed in the forward-scattering hemisphere, while for $x=5$ this was the case in the backward-scattering hemisphere. A similar observation can be made for $-F_{12} / F_{11}$. For $x=5$, the largest differences among different model particles are seen in the angular range $130-170^{\circ}$. By contrast, for $x=50$ both the largest oscillations and the largest differences among different model particles are observed in the forward-scattering hemisphere. Also, $-F_{12} / F_{11}$ is positive for all scattering angles.

\section{Discussion}

\subsection{Computational methods: T-matrix and DDA}

For silicate particles, we found a very good agreement between T-matrix and DDA computations. For hematite, relative differences between the two methods were more pronounced, but absolute differences were small in all cases. Figure 4 (centre left) shows that the T-matrix results obtained for different model geometries are qualitatively very similar; they all show a pronounced minimum around $130^{\circ}$, and much weaker minima around $90^{\circ}$ and $40^{\circ}$. In Fig. 2, the broad and pronounced minima obtained with the DDA deviate from this behaviour. For GRS-2D, Cheb-2D, and Cheb-3D, the accuracy of the T-matrix results were tested by use of the reciprocity condition (e.g. [13, 7]). We found that the maximum allowed error of $5 \%$ was not exceeded in any of the cases studied. In the majority of the cases, the error was even less than $3 \%$. We therefore tend to believe that the T-matrix results are rather reliable. On the other hand, with the serial DDSCAT code it was not possible to obtain convergent results in the hematite case, which is why we had to use the parallel $A D D A$ code with FCD polarizability scheme. This indicates that rough hematite particles with $x=5$ present a numerical challange for the DDA method. The main difficulty is to faithfully 
represent a surface roughness with an angular correlation of only $4^{\circ}$ in a discretised grid. It is also possible that even more dipoles would be needed to accurately reproduce the profile of the electric fields near the particle surface due to the high refractive index. Another possibility would be to change the representation of the polarizability of dipoles that is more suitable for a large refractive index.

These results suggest that the T-matrix approach lends itself more easily to applications of particles with small-scale surface roughness. However, as mentioned earlier, there do exist certain limitations. In T-matrix computations with the null-field method the main source of error is a numerical matrix inversion, which can introduce ill-conditioning problems. Such ill-conditioning problems can be reduced by use of a clever choice of the numerical matrix inversion method [24], such as LU decomposition, or by exploiting irreducible representations of finite groups [12]. The Tsym uses LU decomposition, and it is, to the best of our knowledge, the only T-matrix code that uses irreducible representations of finite groups for reducing the ill-conditioning problems. By this the range of size parameters can be significantly extended. But it is important to realise that the ill-conditioning problems can never be completely eliminated by such methods. In the current study, we avoid the matrix inversion problem by iteratively computing the T-matrix via a Lippmann-Schwinger equation [7]. For spherical particles with a small-scale surface perturbation, this approach entirely circumvents the ill-conditioning problem. However, this method is limited to particles with small perturbation amplitudes. In this study, we performed T-matrix computations for $x=5$ and $x=50$, where the latter has been limited to a deformation parameter $\epsilon=0.01$ (for Chebyshev particles) and an amplitude standard deviation of $\sigma=0.01$ (for Gaussian random spheres). Further, the algorithm for generating GRS-2D and GRS-3D particles can become numerically less accurate at small correlation angles. Numerical inaccuracies in the generation of stochastic particles would not be a major concern in DDA computations. However, in T-matrix computations both the surface parameterisation $r(\theta, \phi)$ and its partial derivatives $\partial r / \partial \theta$ and $\partial r / \partial \phi$ need to be computed. Numerical imprecisions can entail inconsistencies between these quantities, which would introduce errors in the computation of the T-matrix. Our reciprocity tests indicate that the algorithm for generating GRS-2D and GRS-3D particles was numerically reliable down to angular correlations of $\Gamma=4^{\circ}$.

A major limitation in all electromagnetic scattering computations is the 
high CPU-time requirement, which rapidly increases with the particle's size parameter. Besides using non-exact methods, the only known approach for alleviating this problem is to impose symmetry assumptions on the particle geometry, and to systematically exploit these symmetries in the solution of the electromagnetic scattering problem [12]. This excludes the use of irregular geometries with large size parameters. Thus, our T-matrix computations for a size parameter $x=50$ were limited to Cheb-3D, GRS-2D, and Cheb2D geometries, where Cheb-3D of even Chebyshev order $\ell$ belong to a finite symmetry group of order $M_{o}=4 \ell$, and where GRS-2D and Cheb-2D are both axisymmetric, thus of infinite symmetry order. The use of finite symmetry groups reduces CPU-time by a factor of $1 / M_{o}^{2}$. For instance, for the high numerical accuracy we required in this study, the computations for the Cheb-3D particles with $x=50, \ell=240$ took about 1.75 hours. For particles without symmetries, such as GRS-3D, the CPU-time would have been increased by a factor $M_{o}^{2} \sim 10^{6}$ to about 184 years!

\subsection{Model particles: 2D-vs 3D-, and regular vs stochastic roughness models}

It has been reported earlier [1] that the phase function of Cheb-2D particles converges with increasing Chebyshev order. This observation has been confirmed by [2]. Consequently, the optical properties of Cheb-2D particles are, in general, sensitive to the presence of a surface roughness as well as to the roughness amplitude, but they become independent of the perturbation wavelength $\Lambda$ if $\Lambda$ is sufficiently small. One may be tempted to hypothesise that model particles with different representations of surface roughness should yield very similar optical properties, as long as the perturbation wavelength is small. Remarkably enough, this is generally not the case, as the results of this study demonstrate.

We used Chebyshev particles as a proxy for particles with a regular surface roughness, and Gaussian random spheres as a proxy for particles with a stochastic surface roughness. For each of these classes of geometries, we considered 2D and 3D surface perturbations. In general, the optical properties computed with these four classes of geometries agree only in those cases for which the optical properties of rough spheres closely agree with those of smooth spheres, i.e. in those cases in which small-scale surface roughness has a negligible effect on the optical properties. In most cases, the importance of surface roughness increases for larger size parameters and larger perturbation amplitudes. 
Apart from these observations, the results are rather complex, and it is quite difficult to identify any general trends. $F_{11}, \omega$, and $g$ computed for hematite are more sensitve to surface roughness than corresponding results obtained for silicate. This is especially apparent for $x=50$. The opposite is the case for $F_{22} / F_{11}, \delta_{L}$, and $\delta_{C}$. For silicate GRS-2D particles with a size parameter of $x=50, F_{22} / F_{11}, \delta_{L}$, and $\delta_{C}$ are highly sensitive to surface roughness effects, while the corrsponding results for hematite are only weakly sensitive to stochastic surface perturbations. For either refractive index, only stochastic roughness models yield appreciable deviations of $F_{22} / F_{11}$ from unity (the only exception being large hematite Cheb-2D particles). 2D roughness models give larger deviations of $F_{22} / F_{11}$ from unity than $3 \mathrm{D}$ roughness models. But the differences between 2D and 3D roughness models are less pronounced than the differences between stochastic and regular models. The angular range of the differences among various roughness models varies for different $x$ and $m$.

For large hematite particles, regular surface roughness models (Cheb$2 \mathrm{D}$ and Cheb-3D) predict large deviations of $F_{11}$ from the phase function of homogeneous spheres, while stochastic roughness models (GRS-2D and GRS3D) yield results for $F_{11}$ that are rather similar to those obtained for spheres. Note, however, that the computational constraints limited our investigation of large hematite particles $(x=50)$ to very small perturbation amplitudes ( $\epsilon=0.01$ for Chebyshev particles, $\sigma=0.01$ for Gaussian random spheres). Larger perturbation amplitudes would almost certainly give larger deviations of $F_{11}$ computed with model geometries with stochastic surface perturbations. Comparison of the two different classes of Chebyshev particles shows that 2D surface roughness produces a stronger effect in $F_{11}$ than $3 \mathrm{D}$ surface roughness.

The degree of linear polarisation $-F_{12} / F_{11}$ behaves qualitatively similar for both homogeneous spheres and the four classes of rough model particles. The results obtained for different model particles mainly differ in the position and amplitude of the various peaks and troughs of this Mueller matrix element.

The results obtained for GRS particles showed that the optical properties can vary appreciably among different stochastic realisations of the same class of geometry (i.e. among particles with the same angular correlation and amplitude standard deviation). This is especially the case for GRS-2D. It is therefore important to ensemble-average the optical properties when using these model particles. 


\section{Concluding remarks}

Computations of light scattering by particles with small-scale surface roughness proved to be challenging within the discrete dipole approximation. To reproduce the non-vanishing linear depolarisation produced by the surface perturbation, we had to choose a very small dipole spacing in the DDA computations. The T-matrix approach seems to be more versatile for this kind of application. However, T-matrix computations for particles with large size parameters are limited to surface roughness structures with small amplitudes and with at least some finite order of symmetry.

The results presented here clearly show that the significance of small-scale surface roughness strongly depends on the refractive index and the size parameter. The effects of small-scale surface roughness can manifest themselves in different optical properties and, in the case of differential scattering properties, in varying angular ranges. In general, 2D roughness models seem to predict a stronger effect than corresponding 3D roughness models. We found examples in which stochastic models predict more pronounced effects than regular roughness models and vice versa. Thus it is generally not possible to identify any simple patterns for the manifestation of surface-roughness in the scattered intensity and polarisation. These results alert us to interpret modelling studies based on the choice of a specific roughness model with utmost care.

Beyond taking notice of the differences among various roughness models, we are presently not able to decide which model is likely to produce the most accurate results in electromagnetic scattering computations. Constraining models with measurements is likely to be a challenging task. This will require light scattering measurements of rough particles with sufficiently large effective size parameters. Measurable effects on the scattered intensity will be most pronounced for particles with high enough real and imaginary parts of the refractive index. Currently available measurements of the Mueller matrix elements of hematite [25] were performed on a sample with an effective size parameter of $x=4$. As noted in [2], small-scale surface roughness effects for hematite become important at $x \gtrsim 5$. Comparisons of model simulations with measurements may be complicated by the fact that the effects of small-scale surface roughess in mineral particles will be superimposed with the effects of large-scale irregularity and non-sphericity. One way to disentangle these effects may be to consider particles with a very high imaginary part of the refractive index (so that the impact of internal resonances on 
the scattered field is quenched), or to fabricate rough spheres for microwave analog experiments.

Owing to the high computational requirements, our investigation was limited to two size parameters and to two values of the refractive index. There are countless possible extentions of this study. For instance, mineral dust particles in the terrestrial atmosphere typically contain a mass fraction of hematite that varies between $1-4 \%$. This seemingly small range is sufficient to cause significant variations in the imaginary part of the refractive index of mineral aerosols. It would be important to investigate in more detail the extent to which small-scale surface roughness can modulate the optical properties of mineral dust aerosols depending on its hematite content. Another extention of this study would be the evaluation of optical properties of ice cloud particles with rough surfaces (due to riming). This is a very challenging task owing to the large size parameters that need to be considered, especially at visible and near-infrared wavelengths. Finally, it would be interesting to investigate the sensitivity of the optical properties to varying roughness amplitudes.

[1] T. Rother, K. Schmidt, J. Wauer, V. Shcherbakov, J.-F. Gaeyt, Light scattering on Chebyshev particles of higher order, Appl. Opt. 45 (2006) 6030-6037.

[2] M. Kahnert, T. Nousiainen, P. Mauno, On the impact of non-sphericity and small-scale surface roughness on the optical properties of hematite aerosols, J. Quant. Spectrosc. Radiat. Transfer 112 (2011) 1815-1824.

[3] T. Nousiainen, K. Muinonen, P. Räisänen, Scattering of light by Saharan particles in a modified ray-optics approximation, J. Geophys. Res. 108 (2003) oi:10.1029/2001JD001277.

[4] O. Muñoz, H. Volten, J. W. Hovenier, T. Nousiainen, K. Muinonen, D. Guirado, F. Moreno, L. B. F. M. Waters, Scattering matrix of large Saharan dust particles: experiments and computations, J. Geophys. Res. 112 (2007) D13215. doi:10.1029/2006JD008074.

[5] T. Nousiainen, O. Muñoz, H. Lindqvist, P. Mauno, G. Videen, Light scattering by large Saharan dust particles: Comparison of modeling and experimental data for two samples, J. Quant. Spectrosc. Radiat. Transfer 112 (2011) 420-433. doi:10.1016/j.jqsrt.2010.09.003. 
[6] J.-C. Auger, G. Fernandes, K. Aptowicz, Y.-L. Pan, R. Chang, Influence of surface roughness on the elastic-light scattering patterns of micronsized aerosol particles, Appl. Phys. B 99 (2010) 229-234.

[7] M. Kahnert, T. Rother, Modeling optical properties of particles with small-scale surface roughness: combination of group theory with a perturbation approach, Opt. Express 19 (2011) 11138-11151.

[8] T. Nousiainen, K. Muinonen, Surface-roughness effects on singlescattering properties of wavelength-scale particles, J. Quant. Spectrosc. Radiat. Transfer 106 (2007) 389-397. doi:10.1016/j.jqsrt.2007.01.024.

[9] E. Zubko, K. Muinonen, Y. Shkuratov, G. Videen, T. Nousiainen, Scattering of light by roughened Gaussian random particles, J. Quant. Spectrosc. Radiat. Transfer 106 (2007) 604-615.

[10] M. Mishchenko, J. Dlugach, D. Mackowski, Light scattering by wavelength-sized particles dusted with subwavelength-sized grains, Optics Letters 36 (2011) 337-339.

[11] F. M. Kahnert, J. J. Stamnes, K. Stamnes, Application of the extended boundary condition method to homogeneous particles with point group symmetries, Appl. Opt. 40 (2001) 3110-3123.

[12] M. Kahnert, Irreducible representations of finite groups in the T matrix formulation of the electromagnetic scattering problem, J. Opt. Soc. Am. A 22 (2005) 1187-1199.

[13] T. Rother, J. Wauer, Case study about the accuracy behavior of three different T-matrix methods, Appl. Opt. 49 (2010) 5746-5756.

[14] M. I. Mishchenko, Light scattering by randomly oriented axially symmetric particles, J. Opt. Soc. Am. A. 8 (1991) 871-882.

[15] N. G. Khlebtsov, Orientational averaging of light-scattering observables in the T-matrix approach, Appl. Opt. 31 (1992) 5359-5365.

[16] P. C. Waterman, Matrix formulation of electromagnetic scattering, Proc. IEEE 53 (1965) 805-812. 
[17] M. Kahnert, Boundary symmetries in linear differential and integral equation problems applied to the self-consistent Green's function formalism of acoustic and electromagnetic scattering, Opt. Commun. 265 (2006) 383-393.

[18] B. T. Draine, The discrete dipole approximation for light scattering by irregular targets, in: M. I. Mishchenko, J. W. Hovenier, L. D. Travis (Eds.), Light Scattering by Nonspherical Particles, Academic Press, San Diego, 2000, pp. 131-144.

[19] M. A. Yurkin, A. G. Hoekstra, The discrete-dipole-approximation code ADDA: Capabilities and known limitations, J. Quant. Spectrosc. Radiat. Transfer 112 (2011) 2234-2247.

[20] A. Penttilä, E. Zubko, K. Lumme, K. Muinonen, M. A. Yurkin, B. Draine, J. Rahola, A. G. Hoekstra, Y. Shkuratov, Comparison between discrete dipole implementations and exact techniques, J. Quant. Spectrosc. Radiat. Transfer 106 (2007) 417-436.

[21] K. Muinonen, T. Nousiainen, P. Fast, K. Lumme, J. I. Peltoniemi, Light scattering by gaussian random particles: ray optics approximation, J. Quant. Spectrosc. Radiat. Transfer 55 (1996) 577-601.

[22] M. A. Yurkin, M. Min, A. G. Hoekstra, Application of the discrete dipole approximation to very large refractive indices: Filtered coupled dipoles revived, Phys. Rev. E 82 (2011) 036703(12).

[23] J. Tyynelä, E. Zubko, K. Muinonen, G. Videen, Interpretation of singleparticle negative polarization at intermediate scattering angles, Appl. Opt. 49 (2010) 5284-5296.

[24] D. J. Wielaard, M. I. Mishchenko, A. Macke, B. E. Carlson, Improved T-matrix computations for large, nonabsorbing and weakly absorbing nonspherical particles and comparison with geometrical-optics approximation, Appl. Opt. 36 (1997) 4305-4313.

[25] O. Muñoz, H. Volten, J. W. Hovenier, M. Min, Y. G. Shkuratov, J. P. Jalava, W. J. van der Zande, L. B. F. M. Waters, Experimental and computational study of light scattering by irregular particles with extreme refractive indices: hematite and rutile, Astron. Astrophys. 446 (2006) 525-535. 


\section{Acknowledgments}

M. Kahnert acknowledges funding from the Swedish Research Council under project 621-2008-4387 and 621-2011-3346. T. Nousiainen acknowledges the funding by the Academy of Finland (contract 125180). 